\title{
BMJ Open Impact of alprazolam in allostatic load and neurocognition of patients with anxiety disorders and chronic stress (GEMA): observational study protocol
}

\author{
Carlos A Soria, ${ }^{1}$ Carolina Remedi, ${ }^{1}$ Daniel A Núñez, ${ }^{2}$ Luciana D’Alessio, ${ }^{1}$ \\ Emilio J A Roldán ${ }^{2}$
}

To cite: Soria CA, Remedi C, Núñez DA, et al. Impact of alprazolam in allostatic load and neurocognition of patients with anxiety disorders and chronic stress (GEMA): observational study protocol. BMJ Open 2015;5: e007231. doi:10.1136/ bmjopen-2014-007231

- Prepublication history for this paper is available online. To view these files please visit the journal online (http://dx.doi.org/10.1136/ bmjopen-2014-007231)

Received 17 November 2014 Revised 21 May 2015 Accepted 16 June 2015

CrossMark

${ }^{1}$ Department of Córdoba, Institute of Biosciences Henri Laborit, Buenos Aires, Argentina

${ }^{2}$ Department of Scientific Direction, Gador SA, Buenos Aires, Argentina

Correspondence to Dr Luciana D'Alessio; luladalessio@gmail.com

\section{ABSTRACT}

Introduction: The allostatic load model explains the additive effects of multiple biological processes that accelerate pathophysiology related to stress, particularly in the central nervous system. Stressrelated mental conditions such as anxiety disorders and neuroticism (a well-known stress vulnerability factor), have been linked to disturbances of hypothalamo-pituitary-adrenal with cognitive implications. Nevertheless, there are controversial results in the literature and there is a need to determine the impact of the psychopharmacological treatment on allostatic load parameters and in cognitive functions. Gador study of Estres Modulation by Alprazolam, aims to determine the impact of medication on neurobiochemical variables related to chronic stress, metabolic syndrome, neurocognition and quality of life in patients with anxiety, allostatic load and neuroticism.

Methods/analysis: In this observational prospective phase IV study, highly sympthomatic patients with anxiety disorders (six or more points in the Hamilton-A scale), neuroticism (more than 18 points in the Neo five personality factor inventory (NEO-FFI) scale), an allostatic load (three positive clinical or biochemical items at Crimmins and Seeman criteria) will be included. Clinical variables of anxiety, neuroticism, allostatic load, neurobiochemical studies, neurocognition and quality of life will be determined prior and periodically (1, 2, 4, 8, and 12 weeks) after treatment (on demand of alprazolam from $0.75 \mathrm{mg} / \mathrm{day}$ to $3.0 \mathrm{mg} /$ day). A sample of $n=55 / 182$ patients will be considered enough to detect variables higher than $25 \%$ (pretreatment vs post-treatment or significant correlations) with a $1-\beta$ power of $0-80$. $t$ Test and/or non-parametric test, and Pearson's test for correlation analysis will be determined.

Ethics and dissemination: This study protocol was approved by an Independent Ethics Committee of FEFyM (Foundation for Pharmacological Studies and Drugs, Buenos Aires) and by regulatory authorities of Argentina (ANMAT, Dossier \# 61 409-8 of 20 April 2009), following the law of Habeas Data and psychotherapeutic drug control.

Trial registration number: GEMA 20811.
Strengths and limitations of this study

- The mapping of stress pathogenesis is still under construction hence drugs effects needs to be best understood and treatments schedules reconsidered for given patients. Alprazolam effects over its known anxiolysis (anxiolepsis?) is here explored.

- The recruitment of symptomatic patients facilitates the observations of interrelating variables. In order to cut the demand of repeated or protracted drug interventions in chronic stress, neurocognition health is to be preserved. Alprazolam is postulated as a supporting medication in symptomatic subjects.

- The inclusion of patients with an ample range of scoring in each of the study variable facilitates the further observation of the medication impact in patients observed as in daily practice.

- One of the important biases of the project is the lack of a parallel control group, which is not regulatory, allowed in our area in a phase IV trial with highly symptomatic patients with inherent risks. As the project moves forward, however, the project itself can be justified in a more advanced stage, for example, in a face-to-face comparison of different schemes.

- Another difficulty is the existence of knowledge gaps among the clinical features, cognitive observations and variables measured in laboratories. So, we assume that as we move forward in the associations among them, other hypotheses on mechanism of action may arise that can be formulated and tested by other investigators.

\section{INTRODUCTION}

Stress-related mental conditions such as anxiety disorders are frequently associated with chronic stress. ${ }^{1-3}$ If the stress response is excessive and prolonged, the cost of reinstating homeostasis becomes too high, a condition that is termed allostatic load (an indicator of the of adverse health outcomes because of physiological dysregulation). ${ }^{4-10}$ 
Anxiety disorders have been linked to disturbances of hypothalamo-pituitary-adrenal (HPA) axis with controversial results. ${ }^{1-13}$ Hormones, such as cortisol and corticotrophin releasing hormone have been associated with states of fear and anxiety in experimental models. ${ }^{14} 15$ Furthermore, increased basal levels of cortisol were found in patients with panic disorders and in patients with general anxiety disorders (GAD). ${ }^{16}{ }^{17}$ Nevertheless, other authors did not find any association between cortisol levels and anxiety scale measures,${ }^{13}$ or found a reduction in cortisol awakening response among older patients with anxiety disorders. ${ }^{18}$ On the contrary, studies performed among post-traumatic stress disorder patients showed lower levels of cortisol. ${ }^{19}$

Similar patterns of cortisol levels were also found in patients with neuroticism (a predisposition to respond with intense emotional reaction to psychosocial stressors). ${ }^{1}{ }^{2}$ Neuroticism is a well-known stress vulnerability factor that is robustly associated with psychiatric disorders such as anxiety and depression. ${ }^{20}$ Neuroticism and stress domains do not measure an identical construct, nevertheless, it has been reported that cumulative stress was more likely to precipitate psychiatric symptoms in vulnerable individuals with high levels of neuroticism, ${ }^{20}$ and furthermore, these individuals perceive and have more stressors, respond exaggeratedly to them, and require more time to recover from them. ${ }^{12} 21$

During stress activation, both HPA axis and the noradrenaline (NA) system seem to function in a synchronous fashion, and these two systems are crucial for stress response in mammals. ${ }^{6} 7$ There is substantial evidence that anxiety in humans is associated with high levels of 3-methoxy-4-hydroxyphenilglycol (MHPG), a principal NA metabolite, and salivary determination of MHPG may be a useful marker for detecting changes in catecholamine metabolism, ${ }^{22-24}$ however, there is a need to determine, by objective measures, the impact of stress mediators on allostatic load and cognitive functioning in these patient, and the impact of the psychopharmacological treatment on these parameters.

The allostatic load model has been particularly informative in specifying the additive effects of multiple biological processes that contribute to wear and tear on tissues and accelerate pathophysiology related to stress, particularly in the central nervous system. ${ }^{5-7} 2510$ These allodynamic processes can be adaptive in the short term (allostasis) and maladaptive in the long term (allostatic load). The brain is the central organ of stress processes and allodynamic adaptation, and it is a key target of allostasis, thus enabling protection and adaptation. Consequently, allostatic load can affect brain plasticity owing to adjustments made by the various biological systems. ${ }^{9}$

It has been demonstrated that stressful experiences have functionally relevant effects on dendritic arbour and spine/synapse number in many regions of the brain, including the hippocampus, amygdala and the prefrontal cortex (PFC) with effects not only on cognitive function but also on emotional regulation and other self-regulatory behaviours and on neuroendocrine and autonomic function. ${ }^{9}$ By compromising hippocampal function, allostatic load and elevated levels of glucocorticoids impair normal hippocampal function as well as memory processes (declarative memory). ${ }^{7} \quad 26 \quad 27$ Furthermore, stress-related effects on the PFC has an impact in working memory process (the ability to keep events in mind) ${ }^{28}$ and self-regulatory behaviours. The dorsolateral PFC is responsible for planning approaches and sequences of behaviour that are required for goaldirected behaviours. This process is critical of executive function. $^{728}$

In experimental models, chronic restraint stress caused spine loss and debranching of dendrites on PFC neurons and impaired working memory. ${ }^{7} 9^{9} 29$ In humans, adverse childhood experiences were associated with a smaller volume of the PFC, greater activation of the HPA axis, and elevation in inflammation levels. ${ }^{30}$ Furthermore, it has been shown that stress and allostatic load increases interference from irrelevant information, and thus impairs selective attention, ${ }^{31}$ and high levels of cortisol, in response to stressors, showed lower performance in working memory tasks in normal adults. ${ }^{32} \mathrm{Also}$, it has been found that anxiety disorders affect cognitive style of functioning, reversed by alprazolam treatment. ${ }^{33}$

Following the National Institute for Health and Care Excellence, UK, guidelines, treatment of a person with GAD and chronic stress consists of psychotherapy (cognitive behavioural therapy), but when symptoms induce a significant functional impairment, it should be restored with drugs, generally antidepressants and benzodiazepines $^{34}$ (BZD). Benzodiazepines are GABAergic substances with anxiolytic and sedative activity available since the sixties. The therapeutic use to depress acute and subacute symptoms of anxiety disorders has gained great popular acceptance up to the present. ${ }^{35}$ Stress-related mental conditions such as anxiety disorders and neuroticism are treated with psychotherapy and psychopharmacological drugs, which are primarily prescribed to reduce pathological symptoms, but the impact on the underlying disorder is generally less understood. Furthermore, many patients persist with symptoms and consequently require the use of BZDs for longer periods of time. Nevertheless, this indication is an off-label use, and is therefore regarded with caution because of the possibility of favouring dependence on medication and other undesired events such as cognitive impairment. ${ }^{35-37}$ However, when the stressors persist, cognitive variables could be affected, making it difficult for the patient to take decisions that are favourable for quality of life (QOL) ${ }^{3437} 38$

The objective of treatment is not only to suppress symptoms of anxiety, but also to prevent a chronic outcome, and to guide patients to positive behaviours 'decision making' for a better QOL. ${ }^{5}$

As BZDs have anxioliytic and sedating properties, it is expected that they influence the stress system. Also, most 
studies showed a decrease in cortisol levels, ${ }^{39-43}$ some studies reported mixed results, ${ }^{43-45}$ and these inconsistencies may be explained by differences in patient groups, dosages, and BZD used among studies. ${ }^{43}$ Some studies yield a reduction of variations in noradrenaline (NA) metabolite levels (MHPG) 'noradrenergic volatility ${ }^{46}$ and in circulating and salivary cortisol levels using alprazolam in anxious patients ${ }^{22} 3941$ and in normal subjects, ${ }^{47}$ or also an increase in levels of dehydroepiandrosterone (an anticortisol mediator). ${ }^{48}$ Nevertheless, many controversial findings were made depending on age and gender: younger anxious patients showed higher levels of salivary cortisol awakening response, ${ }^{13}{ }^{18}$ but other authors found high salivary cortisol levels associated with severity of late-life generalised anxiety disorders in older adults. ${ }^{17}$ Furthermore, women seem to yield a different profile in the cortisol response to stress in relation to men, on response to alprazolam treatment. ${ }^{42}$ Previous data from our clinical practice showed that the administration of alprazolam results in a fast and significant decrease of MHPG concentrations, and a decrease of noradrenergic volatility accompanied by a slower but equally sustained decrease in salivary cortisol levels ${ }^{49}$ (figure 1).The direct action of alprazolam on GABAergic neurons, and secondarily, on catecholaminergic and serotoninergic neurotransmitters may induce the indirect antistress target reducing cortisol plasma levels. The reduction in 'noradrenergic volatility' defined by the sudden variations in the levels of NA (noradrenaline) and its metabolite MHPG observed in patients with GAD, stress-related diseases and depressive states, may also be involved. ${ }^{46} 50-54$

Nevertheless, the other aspects of benzodiazepines treatment are cognitive adverse effects, and identification of these requires dedicated monitoring of cognitive function, which is often absent from routine hospital and ambulatory clinical care. ${ }^{55}$ The short-term effects of BZD on cognition are well known, and they are

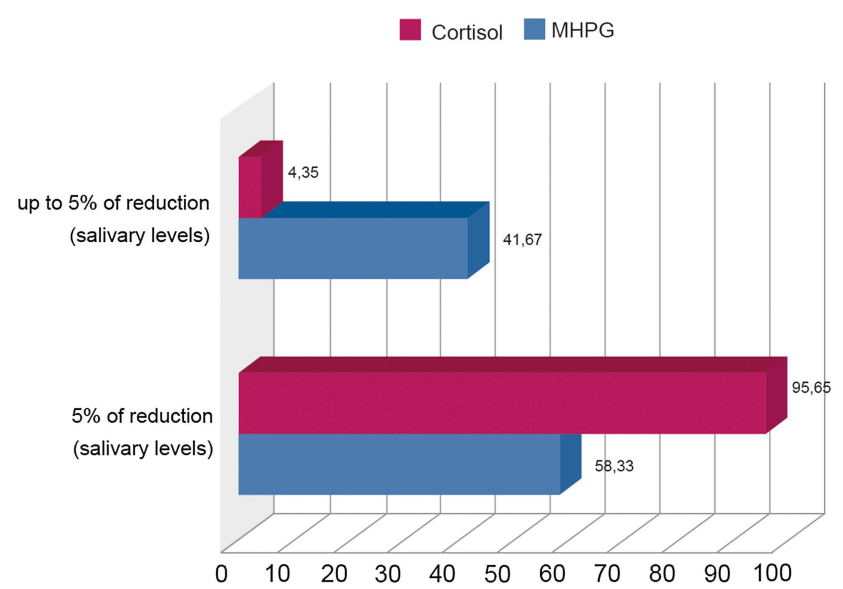

Figure 1 Percentage of reduction of salivary cortisol and 3-methoxy-4-hydroxyphenilglycol (MHPG) levels among alprazolam $(0.75-3.0 \mathrm{mg} /$ day $)$ responder patients. Modified from ref. ${ }^{47}$ mediated through an agonist action on receptors of $\gamma$-aminobutyric acid (GABA A). ${ }^{55}$ A systematic review of the association between benzodiazepines and cognition found that cognitive impairment was presumed to be transient and reversible in most studies. $^{38} 55$ Nevertheless, the long-term adverse effects of BZD on cognition are still debated, ${ }^{36} 55$ and available data shows conflicting results. Some authors found an improvement in memory and an increased risk of dementia in prospective population-based studies, performed in elderly patients with long-term use of BZD. ${ }^{36}{ }^{57-60}$ Furthermore, longer acting BZDs were associated with greater risk of developing dementia compared with shorter acting BZDs. ${ }^{36}$ Nevertheless, other concomitant factors could also be responsible for dementia in such studies. Indeed, a higher degree of neuroticism in midlife was associated with increased risk of dementia and longstanding distress, in a population-based study ${ }^{61}$ Other studies did not show conclusive results, ${ }^{62}{ }^{63}$ or even reported a potential protective effect on behavioural disturbances associated with dementia: the role of GABA in dementia has yet to be fully elucidated, and behavioural symptoms associated with dementia may be a direct or indirect result of GABAergic dysfunction. ${ }^{64} 65$ Furthermore, in young adults, BZD may affect cognition differently depending on gender: cognitive alterations of long-term memory have been reported in women, whereas there was no significant affectation in men. ${ }^{63}$

In this study, we selected alprazolam (a triazolobenzodiazepine) for different reasons: it is a high-potency BZD with an indirect activity through 5 - $\mathrm{HT}_{1 \mathrm{~A}}$ serotonergic receptors, which would produce a regulation of the hyperactive noradrenergic pathways and, through them, a reduction of noradrenergic volatility. The reduction of noradrenergic volatility by high-potency BZDs would have a preventive effect via the noradrenergic system, beyond the central GABAergic anxiolytic effects. The level of concentration and distribution of alprazolam within the CNS is effective when it is administered in relatively low doses, with a differential impact on GABA A receptors, upregulated in individuals under chronic stress, and with less impact on cognitive functions because of the shorter acting time ${ }^{3651606667}$ (table 1).

In the present study, Gador study of Estres Modulation by Alprazolam (GEMA), the following research questions were formulated: how does treatment of anxiety with benzodiazepines impact on allostatic load and neuroticism? Do allostatic load and chronic stress affect cognition process? Do BZDs affect cognitive function positively in stressed patients?

\section{General hypothesis}

In the present study, we aim to investigate biochemical and neurocognitive variables in patients with high anxiety levels associated with chronic stress, and neuroticism (a clinical condition that indicates an exaggerated response to the stressors that require more time to recover from them), ${ }^{51} 6869$ and the impact of 
Table 1 Comparative affinity of different anxiolytics benzodiazepines to GABA A subtypes receptors

\begin{tabular}{|c|c|c|c|c|c|c|}
\hline & \multicolumn{6}{|c|}{ GABA receptor subtype } \\
\hline & $\begin{array}{l}\text { a1 } \\
\text { CNS depression } \\
\text { motor impairment } \\
\text { amnesia }\end{array}$ & $\begin{array}{l}\alpha 2 \\
\text { Anxiolysis } \\
\text { disinhibition }\end{array}$ & $\begin{array}{l}\alpha 3 \\
\text { Anticonvulsive } \\
\text { mio-relaxation } \\
\text { anxiolysis }\end{array}$ & $\begin{array}{l}\alpha 5 \\
\text { Learning } \\
\text { memory }\end{array}$ & $\begin{array}{l}\text { B3 } \\
\text { Anticonvulsive } \\
\text { muscle relaxation } \\
\text { sedation }\end{array}$ & $\begin{array}{l}\text { - } 2 \\
\text { Addiction } \\
\text { respiratory } \\
\text { depression }\end{array}$ \\
\hline Alprazolam & Low & High & Moderate & Low? & Low & High \\
\hline Clonazepam & Moderate & High & High & Low? & Moderate & High \\
\hline Diazepam & Moderate & High & High & Moderate? & Moderate & Moderate \\
\hline Lorazepam & Low & High & High & Moderate? & Low & High \\
\hline
\end{tabular}

intermediate-term treatment (12 weeks) with low doses of alprazolam.

In clinical practice, high symptomatic anxiety disorders are frequently associated with chronic stress and neuroticism (a well-known stress vulnerability factor), ${ }^{20}$ and overall, all these factors increase the vulnerability to diseases and reduce resilience. In these high symptomatic patients, the use of BZDs is required for longer periods of time. Nevertheless, this indication is an offlabel use, and is therefore regarded with caution because of the possibility of favouring dependence on medication and other undesired events such as cognitive functions, reflexes and alert states. ${ }^{35}$

The global hypothesis under investigation is if neurobiochemical indicators of allostatic load (noradrenergic metabolites and cortisol levels) are modulated during treatment with alprazolam, and if there are neurocognitive consequences when treatment maintains neurobiochemical parameters within the normal range. A pharmacological modulation of such mediators may contribute to reduce the impact of allostatic load, promoting resilience and better biochemical and neurocognitive performance translated as a better QOL.

This study is based on the hypothesis that high potency benzodiazepines such as alprazolam may serve as more than single anxiety suppressive agents reducing allostatic load and facilitating adaptative responses. GEMA project proposes an intervention designed to determine if minimum doses of alprazolam adjust neurobiochemical mediators, neurocognition and affect QOL, altered by stress (figure 2).

The question attempted to be solved is, whether anxiolytic pharmacology intervention with alprazolam in these high symptomatic patients modify allostatic load, neurocognition performance and QOL, beyond the known anxiolytic effect.

\section{Objectives (end points)}

The project consists of three complementary stages (figure 3).

- To determine whether clinical and biochemical markers of allostatic load tend to normalise with 12 weeks of therapeutic intervention.

- To determine if neurocognitive performance is modified after treatment, and if there is a positive correlation with biochemical variables of allostatic load and with therapeutic levels.

To assess QOL before and after treatment.

Second, the purpose of this study is to report conditions of failure due to lack of response or emergence of undesired events, including adverse events, and possible situations of habituation to or dependence on alprazolam.

\section{METHODS AND ANALYSIS \\ Protocol design}

The GEMA project, is a prospective phase IV clinical trial. The study began in 2010 and is still undergoing advanced trials. This is a prospective observational study, controlled with on-demand assignment of alprazolam doses in variable doses. It is a phase IV study reported to regulatory authorities in compliance with the rules in force and, consequently, enclosed for the use of experimental controls or interventions.

The presence of variable doses will allow us to establish a continuous range of dose response.

\section{Inclusion criteria}

In this study, highly symptomatic patients over age 21 years, who have at the time of admission anxiety symptoms (six or more points in the Hamilton-A scale), ${ }^{70}$ neuroticism (18 or more points in the Neo five personality factor inventory (NEO-FFI) scale) ${ }^{71}$ allostatic load expressed by at least three positive clinical or biochemical items (Crimmins and Seeman criteria) with normal cognitive performance (25 or more points in the Folstein's Mini-Mental State Examination) ${ }^{72}$ were included. Allostatic load measurement included markers of hypothalmic pituitary axis, sympathetic nervous system, cardiovascular system, metabolic system, renal function, lung function and markers of inflammation. ${ }^{10}$

\section{Exclusion criteria}

Other psychiatric disorders listed under Axis I of the Diagnostic and Statistical Manual of Mental Disorders, Fourth Edition. ${ }^{73}$ There were patients requiring other psychotropics added on to alprazolam, sympathicomimetics, corticosteroids or another medication that might kinetically or dynamically interfere with alprazolam. This 

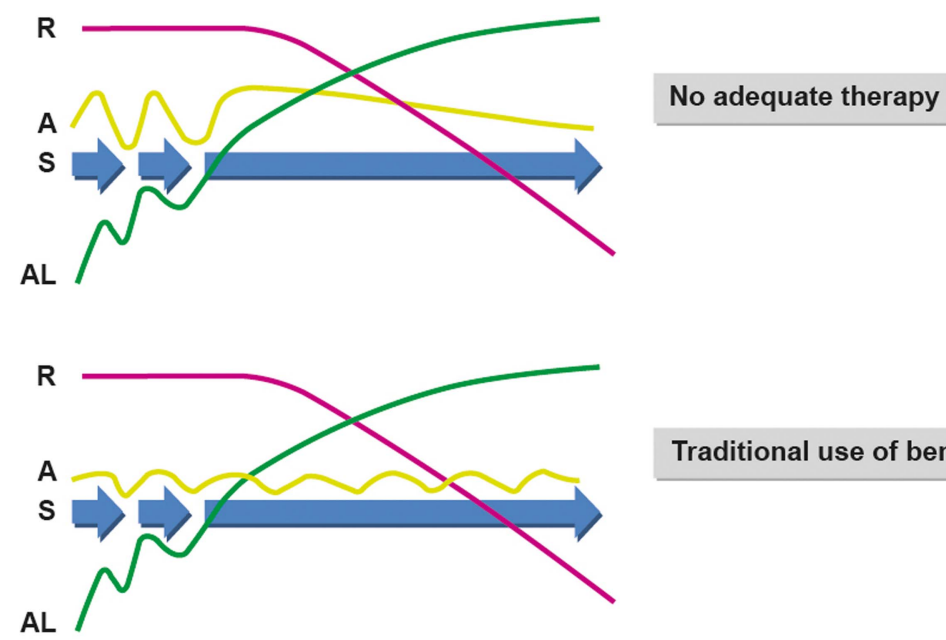

Traditional use of benzodiazepines

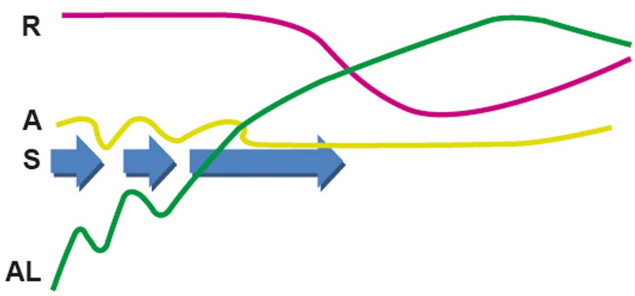

The GEMA project

Figure 2 Upper panel: Repeated stress (S-blue) conditions plus accumulation of allostatic loads (AL-red) result in chronic stress, and with time loss of the individual resilience (R-green) levels. Anxiety (A-yellow) emerges as a reaction of an excessive stress applied to the body. Under chronic stress, cognitive resources are impaired, and with it the possibilities of the affected subject to maintain its quality of life. Consequently, the affected live with a neurobiological environment poorly efficient to solve the daily challenges of life. Middle panel: With a short-term anxiolytic use of alprazolam, symptoms are reduced but demands for treatment persist, as well as the impact of the $\mathrm{AL}$, and the decay of $\mathrm{R}$, are not being significantly modified. Lower panel: With prolonged use of low-dose alprazolam antistress biochemical effects, plus a consequent increase of cognitive functions, may allow the progressive recovering of $\mathrm{R}$, shorten $\mathrm{S}$ duration, and may further reduce the impact of $\mathrm{AL}$. Under these new biological conditions, the subject increased its chances to seek for an improved quality of life, and may therefore shorten the demand of palliative medication.

included patients who had received fluoxetine in the previous 4 weeks, or monoamine oxidase inhibitors in the previous 2 weeks, or showed a history of hypersensitivity to alprazolam. This also included women with confirmed or suspected pregnancy, or persons affected with concomitant conditions, severe or unstable in the cardiovascular system, gastrointestinal, hepatic, renal, neurological and other systems.

Patients will be withdrawn from this study if the dose is prematurely modified, or if the patient decides not to continue with the administered drug, has a related adverse event that modifies his/her daily performance,

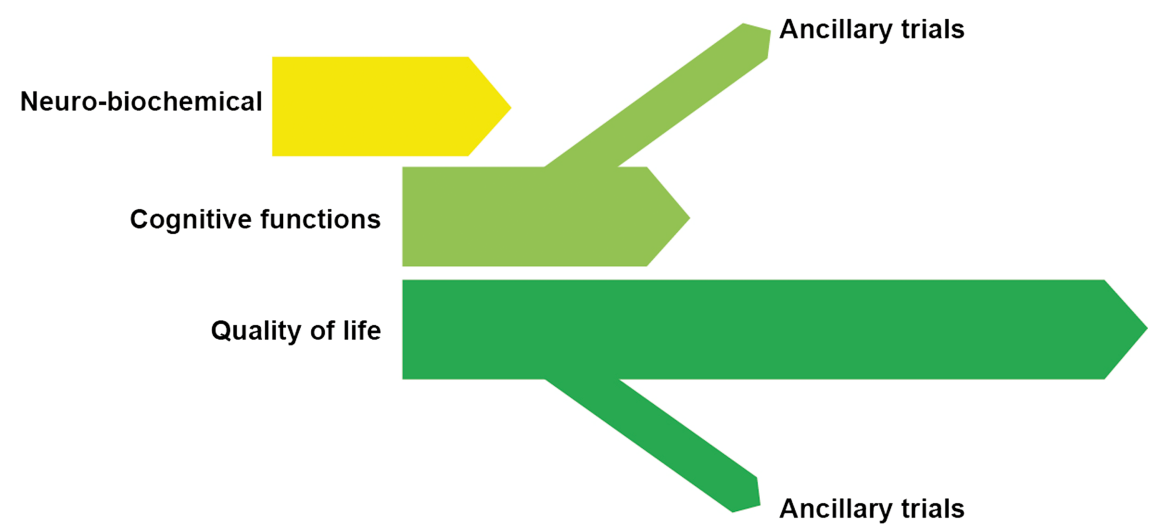

Figure 3 Stage I: Short-term evaluation of $0.75-3.0 \mathrm{mg} /$ day alprazolam on biochemical variables related to chronic stress. Stage II: Short-term evaluation of $0.75-3.0 \mathrm{mg} /$ day alprazolam on cognitive and clinical variables related to chronic stress. Stage III: Intermediate-term evaluation of low alprazolam doses in cognitive, clinical and quality of life indicators (GEMA, Gador study of Estres Modulation by Alprazolam). 
or if the investigator considers that the scheme is not safe or beneficial for the patient according to his/her current clinical status.

\section{Sample size}

Changes in variables higher than $25 \%$ (pretreatment vs post-treatment or significant correlations) with a 1-3 power of $0-80$ are estimated to be detected. For the first phase, $n=29-55$ cases, for the second phase, $n=112$, and for the third an extension of the sample to 182 assessable patients, according to a personal computer (PC) program (Statistica, Tulsa) will be considered enough.

\section{Therapeutic scheme and dose assignment}

Patients will be extensively assessed by a team of medical doctors, clinicians and psychiatrists, who decide the most appropriate approach for each case. The principal investigator will determine the initial dose of alprazolam, usually from 0.75 to $3.0 \mathrm{mg} /$ day, according to the presence of anxiety symptoms and according to patient's tolerance. The ongoing scheme of alprazolam will be maintained for 3 months. Thereafter, the investigator can decide to continue or discontinue treatment gradually, by reducing $0.5 \mathrm{mg}$ every 3 days.

\section{Patient assessment}

Study participants will be evaluated prior to treatment (week 1), on the day treatment started (day 0), and during treatment on weeks $1,2,4,8$ and 12. Clinical variables of anxiety, neuroticism and allostatic load, neurocognitive and neurobiochemical studies will be determined.

Anxiety assessment: Two scales will be rated (1) The Hamilton Anxiety Rating Scale of 14 items. $^{70}$ A $0-5$ score indicates absence of anxiety, 6-14 is mild anxiety, and more than 15 is moderate to severe anxiety. (2) The State-Trait Anxiety Inventory of 40 items, ${ }^{74}$ which assesses the current state and the anxiety trait as a stable tendency. The scale scores a maximum of 60 points, and percentiles are described for gender and age.

Neuroticism assessment: The NEO FFI ${ }^{71}$ is an abbreviated measure of personality factors related to neuroticism, extraversion, openness, agreeableness and conscientiousness. Allostatic load determination: Clinical and biochemical evaluations will be determined by (1) systolic blood pressure will be measured in the supine and standing positions, with a previous 2-3 min rest. A systolic pressure $>140 \mathrm{~mm} \mathrm{Hg}$ and/or a diastolic pressure $<90 \mathrm{~mm} \mathrm{Hg}$ will be considered positive criteria; ${ }^{75}$ (2) heart frequency: a frequency exceeding $90 \mathrm{bpm}$ in supine or standing position with a previous 2-3 min rest is considered a positive pulse; (3) body mass index and waist-hip ratio are measured while the patient is standing and breathing normally, horizontal over the edge of iliac crests, using a tight tape without compressing the skin; (4) laboratory biochemical tests in serum samples include total cholesterol, and high-density lipoprotein and low-density lipoprotein fractions, glycosylated haemoglobin, fibrinogen, albumin, and $\mathrm{C}$ reactive protein. Furthermore, plasma noradrenaline and cortisol and salivary MHPG and cortisol will be determined.

General condition and safety: (1) Clinical Global Impression Scale (National Institute of Mental Health 1976) (CGI), which includes two subscales, (a) clinical profile severity (CGI-S) and (b) clinical profile improvement (CGI-MJ) with eight points in each one. (2) The Mini-Mental State (MEC) quantifies cognitive impairment in time-space orientation, immediate memory, concentration and calculation, long-term memory, language and praxis. ${ }^{72}$ (3) UKU scale (Udvalg fur klinische undersogelser $)^{76}$ to determine side effects; with 56 items: the first 9 are for psychic events, 8 for neurologic, 11 for autonomic and 25 for others. The last two items evaluate side events interference with the patient's daily performance, and their impact on medication intake (none, dose reduction, withdrawal or change). In each one, the investigator assesses whether the event was related to drug treatment or not. (4) Additional safety assessments include respiratory rate and laboratory tests: complete blood count, creatinine, blood urea nitrogen, total bilirubin, alkaline phosphatase, liver enzymes and human chorionic gonadotrophin will be determined when appropriate. All patients will be informed about any eventual depressant effect of alprazolam and risks in activities that require an alert, and the consumption of alcohol will not be recommended. They will also be monitored to detect potential risks of suicide or overdose. All adverse events are monitored, classified and reported according to good clinical practice standards of the local regulatory authority (ANMAT and Ministry of Health of the Province of Córdoba, Argentina).

Cognitive assessment: To quantify the neurocognitive variables; attention, working memory, executive functions, impulsiveness, verbal and non-verbal fluency, the following tests will be conducted: (1) continuous performance test; ${ }^{77}$ (2) digit symbol test; ${ }^{78}$ (3) digit span test: $;^{79}$ (4) verbal fluency test ${ }^{80}$ (5) five point test; ${ }^{81}$ (6) revised Taylor complex test ${ }^{82}$ and (7) Stroop test. ${ }^{83}$ All these tests will be applied after $6 \mathrm{~h}$ since the last administration of alprazolam (nadir plasma levels). Cognitive tests and laboratory tests are blinded to the analyst, while the remaining tests are open.

QOL assessment: The health and QOL scale, health questionnaire SF-36 validated Spanish version will be determined. ${ }^{84}$

\section{Statistical assessment}

The mean, minimum and maximum values of quantitative variables will be determined. Pretreatment and posttreatment values will be compared using $\mathrm{t}$ test and/or a non-parametric test. Statistical significance will be fixed at $\mathrm{p}<0.05$ (2-sided; $\beta-1$ power $\geq 0.80$ ). Correlation analysis will be performed by calculating the Pearson Test correlation coefficient for ungrouped data; with $r>0.80$ and $\mathrm{p}<0.05$ (2-sided) strong correlation, and $0.80<\mathrm{r}>0.50$ and $\mathrm{p}<0.05$ (2-sided), moderate correlation. 
Treatment safety: Regarding patient safety, the use of alprazolam, a well-known, low action, nervous system depressant drug, at minimum doses, even when no symptoms palliation is required, guarantees good tolerability, and no drawbacks are expected as a result of direct effects. It is worth emphasising that the proposed intervention tends to reduce long and/or very frequently repeated demands for medication, and with that improve the global safety rate and QOL.

\section{ETHICS AND DISSEMINATION: APPROVAL AND INFORMED CONSENT}

All participants will need to agree voluntarily to participate, after having obtained informed consent and signed it, in accordance with local regulations and standards of bioethics. The study is developed under phase IV conditions, but the full therapeutic intervention has no cost for the patient under the study protocol. The present study protocol is still ongoing and patient recruitment is in process. Trial Registration: GEMA20811.

\section{EXPECTED RESULTS}

It is our expectation to see a decline within anxiety scores and allostatic load reflected by biochemical variables, and an improvement in cognitive performance with a better QOL, even in patients treated with low doses of alprazolam. We expect to elucidate improvements in cognitive variables, such as time-space orientation, immediate memory, concentration and calculation, long-term memory, language and praxis, as well as a tendency to an overall improvement of QOL scores. If the GEMA project fulfils its basic purposes, a new pathway would open for the search of specific modulators for the treatment of mental illnesses. It has been stated by health economists that the 'macro' conditions of the cost/health ratio are given more by classic or vintage drugs rather than by innovations, many of which disappear after a promotional period. In this sense, alprazolam has persisted as a medical tool for several years, and regardless of what formal pharmaceutical clinical trials and the data that investigators might have to justify their permanence. The GEMA project is also an update in the knowledge of the impact of this compound for patients with anxiety and allostatic load, and is also a project designed to define a new profile for the biomedical use of alprazolam.

\section{DISCUSSION}

The GEMA project constitutes an observational clinical trial designed to determine objective measures of stress pathophysiology in high symptomatic patients with comorbid anxiety, allostatic load and neuroticism treated with minimum doses of alprazolam, with the aim to determine neurobiochemical and neuropsychological variables altered by stress.
Treatment aims to contribute to prevent the impact of allostatic load, promoting resilience, better cognitive performance and making healthier decisions translated into a better QOL. If the hypothesis is confirmed, a new paradigm would come into being, which would renew interest in the clinical management of these compounds beyond their typical use as anxiolytic agents. In short, the proposal is to treat each episode more efficiently in order to reduce the time required to achieve positive results (bene facere), while decreasing the tendency to excessive consumption of drugs (non nocere).

Acknowledgements The authors are grateful for the participation and contributions of the work team involved in GEMA.

Contributors CAS, CR, DAN and EJAR have made substantial contributions to conception and design. LD'A has been involved in drafting and revising the manuscript for intellectual content. EJAR has given final approval of the version to be published.

Funding This work is carried out under a grant awarded by Gador SA, Buenos Aires (2010-2012) and funds of the Instituto de Biociencias Henri Laborit, Córdoba.

Competing interests CAS and LD are consultants at Gador SA, and DAN and EJAR are investigators employed by Gador SA, and CR does not declare any conflict of interest. Gador SA, Darwin 429, Buenos Aires City, Argentina.

\section{Patient consent Obtained.}

Ethics approval The protocol was approved by an Independent Ethics Committee of FEFyM (Fundación de Estudios Farmacológicos y Medicamentos/Foundation for Pharmacological Studies and Drugs, Buenos Aires), and by regulatory authorities of Argentina (ANMAT, Dossier \# 61409-8 of 20 April 2009), following the law of Habeas Data and psychotherapeutic drug control.

Provenance and peer review Not commissioned; externally peer reviewed.

Open Access This is an Open Access article distributed in accordance with the Creative Commons Attribution Non Commercial (CC BY-NC 4.0) license, which permits others to distribute, remix, adapt, build upon this work noncommercially, and license their derivative works on different terms, provided the original work is properly cited and the use is non-commercial. See: http:// creativecommons.org/licenses/by-nc/4.0/

\section{REFERENCES}

1. Lahey BB. Public health significance of neuroticism. Am Psychol 2009;4:241-56.

2. Garcia-Banda G, Chellew K, Fornes J, et al. Neuroticism and cortisol: pinning down an expected effect. Int $J$ Psychophysiol 2014;91:132-8.

3. Plag J, Gaudlitz K, Schumacher S, et al. Effect of combined cognitive-behavioural therapy and endurance training on cortisol and salivary alpha-amylase in panic disorder. J Psychiatr Res 2014;58:12-19.

4. Mc Ewen BS. Stress, adaptation, and disease. Allostasis and allostatic load. Ann N Y Acad Sci 1998;1:33-44.

5. McEwen BS. Stressed or stressed out: what is the difference? J Psychiatry Neurosci 2005;30:315-18.

6. McEwen BS. Effects of adverse experiences for brain structure and function. Biol Psychiatry 2000;48:721-31.

7. Karatsoreos IN, McEwen BS. Resilience and vulnerability: a neurobiological perspective. F1000Prime Rep 2013;5:13.

8. Brotman DJ, Golden SH, Wittstein IS. The cardiovascular toll of stress. Lancet 2007;370:1089-100.

9. McEwen BS, Gianaros PJ. Stress- and allostasis-induced brain plasticity. Annu Rev Med 2011;62:431-45.

10. Crimmins EM, Johnston $M$, Hayward $M$, et al. Age differences in allostatic load: an index of physiological dysregulation. Exp Gerontol 2003;38:731-4.

11. Maidana $P$, Bruno OD. Medición de cortisol y sus fracciones: Concepto de fracción activa. Medicina 2013;73:579-84. 
12. Schumacher S, Gaudlitz K, Plag J, et al. Who is stressed? A pilot study of salivary cortisol and alpha-amylase concentrations in agoraphobic patients and their novice therapists undergoing in vivo exposure. Psychoneuroendocrinology 2014;49:280-9.

13. Heaney JLJ, Phillips AC, Carroll D. Ageing, depression, anxiety, social support and the diurnal rhythm and awakening response of salivary cortisol. Int J Psychophysiol. 2010;78:201-8.

14. Davis M, Walker DL, Lee Y. Amygdala and bed nucleus of the stria terminalis: differential roles in fear and anxiety measured with the acoustic startle reflex. Philos Trans $R$ Soc Lond B Biol Sci 1997;352:1675-87.

15. Sanford LD, Nassar P, Ross RJ, et al. Prolactin microinjections into the amygdalar central nucleus lead to decreased NREM sleep. Sleep Res Online 1998;1:109-13.

16. Wedekind D, Bandelow B, Broocks A, et al. Salivary, total plasma and plasma free cortisol in panic disorder. $J$ Neural Transm 2000;107:831-7.

17. Mantella RC, Butters M, Amico J, et al. Salivary cortisol is associated with diagnosis and severity of late-life generalized anxiety disorder. Psychoneuroendocrinology 2008;33:773-81.

18. Hek K, Direk N, Newson RS, et al. Anxiety disorders and salivary cortisol levels in older adults: a population-based study. Psychoneuroendocrinology 2013;38:300-5.

19. Yehuda R. Advances in understanding neuroendocrine alterations in PTSD and their therapeutic implications. Ann N Y Acad Sci 2006;1071:137-66.

20. Vinkers $\mathrm{CH}$, Joëls $\mathrm{M}$, Milaneschi $\mathrm{Y}$, et al. Stress exposure across the life span cumulatively increases depression risk and is moderated by neuroticism. Depress Anxiety 2014;31:737-45.

21. Suls J, Martin RJ. The daily life of the garden-variety neurotic: reactivity, stressor exposure, mood spillover, and maladaptive coping. J Pers 2005;73:1485-509.

22. Yamada S, Yamauchi K, Yajima J, et al. Saliva level of free 3-methoxy-4-hydroxyphenylglycol (MHPG) as a biological index of anxiety disorders. Psychiatry Res 2000;93:217-23.

23. Karege F, Bovier P, Gaillard J, et al. The heterogeneity of 3 -met hoxy-4-hydr oxy- phenylglycol levels among depressed patients. Acta Psychiatr Sacand 1989;80:499-504.

24. Muscettola G, Potter WZ, Pickar D, et al. Urinary 3-methoxy-4-hydroxyphenylglycol and major affective disorders. A replication and new findings. Arch Gen Psychiatry 1984;41: 337-42.

25. Seeman T, Epel E, Gruenewald T, et al. Socio-economic differentials in peripheral biology: cumulative allostatic load. Ann N Y Acad Sci 2010;1186:223-39.

26. Sheline YI, Wang PW, Gado MH, et al. Hippocampal atrophy in recurrent major depression. Proc Natl Acad Sci USA 1996;93:3908-13.

27. Sheline $\mathrm{YI}$, Sanghavi M, Mintun M, et al. Depression duration but not age predicts hippocampal volume loss in medically healthy women with recurrent major depression. J Neurosci 1999;19:5034-43.

28. Bergson C, Mrzljak L, Smiley F, et al. Regional, cellular, and subcellular variations in the distribution of D1 and D5 dopamine receptors in primate brain. J Neurosci 1995;15:7821-36.

29. Hains $A B, V u$ MAT, Maciejewski PK, et al. Inhibition of protein kinase $\mathrm{C}$ signaling protects prefrontal cortex dendritic spines and cognition from the effects of chronic stress. Proc Natl Acad Sci USA 2009;106:17957-62.

30. Danese A, McEwen BS. Adverse childhood experiences, allostasis, allostatic load, and age-related disease. Physiol Behav 2012;106:29-39.

31. Braunstein-Bercovitz H, Dimentman-Ashkenazi I, Lubow RE. Stress affects the selection of relevant from irrelevant stimuli. Emotion 2001;1:182-92

32. al'Absi M, Hugdahl K, Lovallo WR. Adrenocortical stress responses and altered working memory performance. Psychophysiology 2002;39:95-9.

33. Uhlenhuth EH, Starcevic V, Qualls C, et al. Cognitive style, alprazolam plasma levels, and treatment response in panic disorder. Depress Anxiety 2008;25:E18-26.

34. Hoge EA, Ivkovic A, Fricchione GL. Generalized anxiety disorder: diagnosis and treatment. BMJ 2012;345:e7500.

35. Woods JH, Katz JL, Winger G. Benzodiazepines: use, abuse, and consequences. Pharmacol Rev 1992;44:151-347.

36. Billioti de Gage S, Bégaud B, Bazin F, et al. Benzodiazepine use and risk of dementia: prospective population based study. BMJ 2012;345:e6231.

37. Barker MJ, Greenwood KM, Jackson M, et al. Persistence of cognitive effects after withdrawal from long-term benzodiazepine use: a meta-analysis. Arch Clin Neuropsychol 2004;19:437-54.
38. Wein AJ. A systematic review of amnestic and non-amnestic mild cognitive impairment induced by anticholinergic, antihistamine, GABAergic and opioid drugs. J Urol 2013;190:2168.

39. Curtis GC, Abelson JL, Gold PW. Adrenocorticotropic hormone and cortisol responses to corticotropin-releasing hormone: changes in panic disorder and effects of alprazolam treatment. Biol Psychiatry 1997:41:76-85.

40. Abelson JL, Curtis GC, Cameron OG.

Hypothalamic-pituitary-adrenal axis activity in panic disorder: effects of alprazolam on $24 \mathrm{~h}$ secretion of adrenocorticotropin and cortisol. J Psychiatr Res 1996;30:79-93.

41. Fries $\mathrm{E}$, Hellhammer $\mathrm{DH}$, Hellhammer J. Attenuation of the hypothalamic-pituitary-adrenal axis responsivity to the Trier Social Stress Test by the benzodiazepine alprazolam. Psychoneuroendocrinology 2006:31:1278-88.

42. Pomara N, Willoughby LM, Ritchie JC, et al. Sex-related elevation in cortisol during chronic treatment with alprazolam associated with enhanced cognitive performance. Psychopharmacology 2005;182:414-19.

43. Manthey L, Giltay EJ, van Veen T, et al. Long-term benzodiazepine use and salivary cortisol: the Netherlands Study of Depression and Anxiety (NESDA). J Clin Psychopharmacol 2010;30:160-8.

44. Laakmann $\mathrm{G}$, Wittmann M, Gugath $\mathrm{M}$, et al. Effects of psychotropic drugs (desimipramine, chlorimipramine, sulpiride and diazepam) on the human HPA axis. Psychopharmacology 1984;84:66-70.

45. Pomara N, Willoughby LM, Ritchie JC, et al. Interdose elevation in plasma cortisol during chronic treatment with alprazolam but not lorazepam in the elderly. Neuropsychopharmacology 2004;29:605-11.

46. Coplan JD, Papp LA, Pine D, et al. Clinical improvement with fluoxetine therapy and noradrenergic function in patients with panic disorder. Arch Gen Psychiatry 1997;54:643-8.

47. Zemishlany Z, McQueeney R, Gabriel SM, et al. Neuroendocrine and monoaminergic responses to acute administration of alprazolam in normal subjects. Neuropsychobiology 1990;23:124-8.

48. Kroboth PD, Salek FS, Stone RA, et al. Alprazolam increases dehydroepiandrosterone concentrations. J Clin Psychopharmacol 1999;19:114-24.

49. Soria CA, Remedi C, Muñóz MS, et al. Translational assessment of alprazolam on MHPG, cortisol and cognitive function in patients with neuroticism, allostatic load and anxiety disorders. Int $J$ Neuropsychopharmacol 2010;13:268.

50. Möhler H. Role of GABAA receptors in cognition. Biochem Soc Trans 2009;37:1328-33.

51. Maubach K. GABA(A) receptor subtype selective cognition enhancers. Curr Drug Targets-CNS Neurol Disord 2003;2:233-9.

52. Vasa RA, Pine DS, Masten CL, et al. Effects of yohimbine and hydrocortisone on panic symptoms, autonomic responses, and attention to threat in healthy adults. Psychopharmacology 2009;204:445-55

53. Wong M, Kling MA, Munson PJ, et al. Pronounced and sustained central hypernoradrenergic function in major depression with melancholic features: relation to hypercortisolism and corticotropin-releasing hormone. Clin Res 2000;97:325-30.

54. Juster R, Mcewen BS, Lupien SJ. Neuroscience and Biobehavioral Reviews Allostatic load biomarkers of chronic stress and impact on health and cognition. Neurosci Biobehav Rev 2010;35:2-16.

55. Yaffe K, Boustani M. Benzodiazepines and risk of Alzheimer's disease. BMJ 2014;349:g5312.

56. Curran HV. Tranquillising memories: a review of the effects of benzodiazepines on human memory. Biol Psychol 1986;23:179-213.

57. Hanlon JT, Horner RD, Schmader KE, et al. Benzodiazepine use and cognitive function among community-dwelling elderly. Clin Pharmacol Ther 1998;64:684-92.

58. Paterniti S, Dufouil CAA. Long-term benzodiazepine use and cognitive decline in the elderly: the Epidemiology of Vascular Aging Study. J Clin Psychopharmacol 2002;22:285-93.

59. Gallacher J, Elwood P, Pickering J, et al. Benzodiazepine use and risk of dementia: evidence from the Caerphilly Prospective Study (CaPS). J Epidemiol Community Heal 2012;66:869-73.

60. Wu CS, Wang SC, Chang IS, et al. The association between dementia and long-term use of benzodiazepine in the elderly: nested case-control study using claims data. Am J Geriatr Psychiatry 2009:17:614-20.

61. Johansson L, Duberstein PR, Hällström T. Midlife personality and risk of Alzheimer disease and distress. Neurology 2015;84:1526-632.

62. Allard J, Artero S, Ritchie K. Consumption of psychotropic medication in the elderly: a re-evaluation of its effect on cognitive performance. Int J Geriatr Psychiatry 2004;18:874-8. 
63. Boeuf-Cazou O, Bongue B, Ansiau D, et al. Impact of long-term benzodiazepine use on cognitive functioning in young adults: the VISAT cohort. Eur J Clin Pharmacol 2011;67:1045-52.

64. Fastbom J, Forsell Y, Winblad B. Benzodiazepines may have protective effects against Alzheimer disease. Alzheimer Dis Assoc Disord 1998;12:14-17.

65. Sultan S, Gebara EG, Moullec K, et al. D-serine increases adult hippocampal neurogenesis. Front Neurosci 2013;7:155

66. Ellinwood EH, Heatherly DG, Nikaido AM, et al. Comparative pharmacokinetics and pharmacodynamics of lorazepam, alprazolam and diazepam. Psychopharmacology (Berl) 1985;86: 392-9.

67. Tan KR, Rudolph U, Lüscher C. Hooke don benzodiazepines: GABAA receptor subtypes and addiction. Trends in Neurosci 2011;34:188-97.

68. Norton PJ, Price EC. A meta-analytic review of adult cognitive-behavioral treatment outcome across the anxiety disorders. J Nerv Ment Dis 2007;195:521-31.

69. Fleishaker JC, Garzone PD, Chambers JH, et al. Comparison of the spectrum of cognitive effects of alprazolam and adinazolam after single doses in healthy subjects. Psychopharmacology 1995;120:169-76.

70. Hamilton M. Hamilton anxiety rating scale (HAM-A). Br J Med Psychol 1959;32:50-5.

71. Costa PT, McCrae RR. Inventario de Personalidad NEO revisado (NEO PI-R) e Inventario NEO reducido de Cinco Factores (NEO-FFI). Manual Professional. Madrid: TEA Ediciones, 1999.

72. Folstein MF. Mini-Mental State: a practical method for grading the mental state of patients for the clinician. Psychiatr Res 1975;12:189-98.
73. American Psychiatric Association. Diagnostic and statistical manual of mental disorders: DSM-IV. 4th edn. Washington DC: American Psychiatric Association, 1994.

74. Laux L, Glanzmann P, Schaffner P, et al. State-Trait-Angst-Inventar STAl. Weinheim: Beltz, 1981.

75. WHO. Mental disorders glossary and guide to their classification in accordance with the ninth revision of the international classification of diseases. Geneva: WHO, 1978.

76. Lingjaerde $\mathrm{O}$, Ahlfors UG, Bech $\mathrm{P}$, et al. The UKU side effect rating scale. A new comprehensive rating scale for psychotropic drugs and a cross-sectional study of side effects in neuroleptic-treated patients. Acta Psychiatr Scand Suppl 1987;334:1-100.

77. Conners K, Jeff JL. $A D H D$ in adults and children: The latest assessment and treatment strategies. Kansas City, MO: Compact Clinicals, 1999.

78. Wechsler D. Manual, Wechsler Adult Intelligence Scale—revised. Psychol Corp New York. 1981.

79. Wechsler D. WMS-III Administration and Scoring Manual. San Antonio, TX: The Psychological Corporation. Harcourt Brace \& Co., 1997.

80. Elwood ER. The Wechsler Memory Scale-Revised: Psychometric characteristics and clinical application. Neuropsychology Review 1991;2:179-201.

81. Regard M, Strauss E, Knapp P. Childrens' production on verbal and non-verbal fluency tasks. Percept Mot Ski 1982;55:839-44.

82. Taylor LB. Localization of cerebral lesions by psychological testing Clin Neurosurg 1969;16:269-87.

83. Stroop JR. Studies of interference in serial verbal reactions. J Exp Psychol 1935;18:643-62.

84. Augustovskia FA, Lewina G, García Elorriob E. The ArgentineSpanish SF-36 Health Survey was successfully validated for local outcome research. J Clin Epidemiol 2008;61:1279-84. 


\section{Correction}

Soria CA, Remedi C, Núnez DA, et al. Impact of alprazolam in allostatic load and neurocognition of patients with anxiety disorders and chronic stress (GEMA): observational study protocol. BMJ Open 2015;5:e007231. There is an error in figure 2 legend of this manuscript. The correct figure legend is:

Figure 2 Upper panel: Repeated stress (S-blue) conditions plus accumulation of allostatic loads (AL-green) result in chronic stress, and with time loss of the individual resilience (R-red) levels. Anxiety (A-yellow) emerges as a reaction of an excessive stress applied to the body. Under chronic stress, cognitive resources are impaired and with it the possibilities of the affected subject to maintain its quality of life. Consequently, the affected live with a neurobiological environment poorly efficient to solve the daily challenges of life. Middle panel: With a short-term anxiolytic use of alprazolam, symptoms are reduced but demands of treatment persist, as well as the impact of the AL, and the decay of R, are not being significantly modified. Lower panel: With prolonged use of low-dose alprazolam anti-stress biochemical effects, plus a consequent increase of cognitive functions, may allow the progressive recovering of $\mathrm{R}$, shorten $\mathrm{S}$ duration, and may further reduce the impact of AL. Under these new biological conditions the subject increased its chances to seek for an improved quality of life and may thereof shorten the demand of palliative medication.

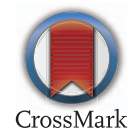

BMJ Open 2015;5:e007231. doi:10.1136/bmjopen-2014-007231corr1 